\author{
Dr. Julio Yarmuch \\ Editor Jefe \\ Presente
}

\section{Sinceramiento de la cirugía bariátrica desde el ombligo del mundo}

El Centro Integral de Nutrición y Obesidad CINTO de Clínica Tabancura, ha querido implementar un proyecto donde poder exponer lo que creemos que debe ser una óptima relación entre los cirujanos; de esta manera aportar a una mejor forma de hacer las cosas, en beneficio de los pacientes.

En el año 2007, los Drs. Cristián Ovalle Letelier y Carlos Farías Ortega, percibieron la necesidad de crear una instancia totalmente diferente de acercamiento entre los Cirujanos Bariátricos de Arica a Punta Arenas.

La base de esta percepción residía en lograr "Sincerar" esta especialidad, más allá del rol médico, dándole énfasis a la apertura como personas.

La responsabilidad de concretar dicha idea recayó en la Psicóloga Siomara Chahuán Chahuán y en la Relatora Marcela Galleguillos Poblete, quienes levantaron un proyecto destinado a acercar a diferentes profesionales que estuvieran realizando Cirugía Bariátrica desde un punto de vista humano, personal y no técnico (como todos los realizados hasta hoy).

De ahí nace como objetivo central lo que se conoce como el Sinceramiento, es decir, que los profesionales compartieran en un medio relajado y distendido, en forma franca y abierta, sus éxitos, fracasos y forma de abordar los problemas.

Hasta hoy no era común hablar de la persona detrás del cirujano, de qué piensa de sus pacientes y de sus colegas. De cómo cree el que es visto por los demás (humanamente hablando) y de cuáles son los temas que debieran tratarse entre los diferentes prestadores, respetando la sana competencia.

No se trataba simplemente sólo de conversar e intercambiar ideas. Se quería ir un paso más allá.

Es así como las profesionales diseñaron dinámicas especialmente pensadas como herramientas para hacer un trabajo interno de Desarrollo Personal.

¿Quiénes eran?, ¿Cómo pensaban acerca de distintos aspectos de la vida?... era como sacarles "el delantal y el bisturí" para comenzar una apertura que les permitiera conocerse como personas, sin dejar el norte que los convocaba: obtener el enriquecimiento colectivo que redundara en el mayor bienestar de los pacientes y en una mejor forma de ser "colegas".
Era ir de un plano personal a uno profesional, potenciando la forma de comunicarse entre ellos.

El proyecto destinó días de trabajo para que, luego de las actividades y de las dinámicas, fueran madurando ideas y opiniones, internalizando en forma lenta pero firme esta nueva forma de trabajo. A esta apertura se le denominó Base de Confianza.

Se determinó que para lograr los objetivos propuestos, el lugar del encuentro debía ser de interés común, nuevo, interesante, entretenido y exótico, para entusiasmar, y lo suficientemente alejado para evitar la distracción.

Rapa Nui cumplía con todos los requisitos, además de aportar una cultura étnica ancestral, basada en el respeto, la confianza y la verdad.

Para la elección de los integrantes de cada grupo, se consideraron varios aspectos:

- Ser miembro del Departamento Bariátrico de la Sociedad de Cirujanos de Chile.

- Estar dedicado principalmente en su quehacer quirúrgico a la cirugía de la obesidad.

- Ser referente de su hospital, clínica u organización.

- Estar dispuesto a destinar un tiempo exclusivo para esta actividad.

- Guardar un equilibrio entre los integrantes, de tal manera que hubiera profesionales de diferentes regiones de Chile.

Los resultados de las dinámicas construidas especialmente para ellos (primer y segundo grupo), dieron los frutos esperados:

- Confianza en el proyecto sin conocer su contenido.

- Se logró el Sinceramiento y la Base de Confianza, ya que hoy en día los profesionales se comunican entre ellos para pedir ayuda, consejo, opinión y hasta derivación de pacientes. Se produjo una apertura individual y grupal, por medio de una conversación espontánea y libre, acerca de sus pensamientos, emociones, éxitos y fracasos.

- Se dio la apertura en el desarrollo personal, demostrado a través de la fraternidad, y la relación honesta, vislumbrándose facetas cálidas y humanas entre ellos.

- La creación y el logro de los objetivos de cada una de las dinámicas y actividades recreativas con cada grupo de profesionales cimentó las raíces para, de común acuerdo, materializar las ideas en hechos concretos, como lo son la creación, a futuro, de una página web de Cirugía Baríatrica, la unificación del consentimiento informado y 
de un informe médico de Cirugía Bariátrica para las Isapres. De igual forma nació la idea de crear una beca de la Universidad de Chile, al mejor alumno de Rapa Nui, con el compromiso de realizar una beca en Medicina Familiar y volver a ejercer a la Isla como médico.

A futuro se espera que todo el Departamento de Cirugía Bariátrica pase por esta experiencia y que la Base de confianza siga trabajando, con un constante refuerzo de los logros obtenidos en la Isla de Pascua, tanto profesional como grupalmente con actividades como éstas, destinadas a aumentar la cohesión y el compromiso de los profesionales.

Nosotras, como profesionales, nos sentimos honradas de la confianza depositada para realizar este proyecto, ya que no existe en el medio médico una experiencia similar de tal magnitud y éxito.

Esperamos que Rapa Nui cada año nos abra las puertas para que más profesionales de la salud pasen por este proceso.

Siomara Chahuán Ch. ${ }^{1}$, Marcela Galleguillos P. ${ }^{2}$ ${ }^{1}$ Psicóloga Clínica y Forense, ${ }^{2}$ Relatora - Relacionadora Pública, Clínica Tabancura, Santiago, Chile.

Profesionales que formaron parte del primer grupo de encuentro en Isla de Pascua 2007:

Dr. José Ignacio Amat.

Clínica Dávila.

Dr. William Awad.

Integramédica.

Dr. Marcos Berry.

Director del Departamento de Cirugía Bariátrica.

Clínica Las Condes

Dr. Patricio Burdiles.

Hospital Clínico Universidad de Chile José Joaquín Aguirre

Dr. Carlos Carvajal.

Clínica Las Condes.

Dr. Attila Csendes.

Hospital Clínico Universidad de Chile José Joaquín Aguirre

Dr. Carlos Farías.

Clínica Tabancura.

Dr. Federico Hernández.

Hospital Barros Luco.
Dr. Luis Ibáñez.

Hospital Clínico Universidad Católica.

Dr. Giulio Innocenti.

Clínica Dávila y Clínica Tabancura.

Dr. Cristián Ovalle.

Clínica Tabancura.

Dr. Félix Raimann

Clínica Alemana de Puerto Varas.

Dr. Domingo Todorovic.

Clínica Dávila.

Dr. Jorge Widerstrom.

Hospital de Iquique.

Cirujanos que formaron parte del segundo grupo de encuentro en Isla de Pascua 2008:

Dr. Patricio Burdiles

Director Departamento de Cirugía Bariátrica.

Clínica Las Condes.

Dr. Juan Eduardo Contreras.

Clínica Santa María.

Dr. Jorge Cárcamo.

Punta Arenas.

Dr. Carlos Cárcamo.

Valdivia.

Dr. Carlos Farías.

Clínica Tabancura.

Dr. Sergio Guzmán.

Hospital Clínico Universidad Católica.

Dr. Fernando Maluenda.

Clínica Alemana.

Dr. Cristián Martínez.

Hospital Sótero del Río.

Dr. Cristián Ovalle.

Clínica Tabancura.

Dr. Francisco Pacheco.

Concepción.

Dr. Alvaro Tapia.

Hospital de Talca.

Dr. Marco Antonio Vargas.

Hospital San José.

Dr. Julio Yarmuch.

Hospital Clínico Universidad de Chile José Joaquín Aguirre. 